\title{
Age-Related Differences in Search Strategy and Performance When Using a Data-Rich Web Site
}

\author{
Erica Olmsted-Hawala ${ }^{1, *}$, Jennifer C. Romano Bergstrom ${ }^{2}$, and Wendy A. Rogers ${ }^{3}$ \\ ${ }^{1}$ U.S. Census Bureau, Center for Survey Measurement, 4600 Silver Hill Road, \\ Washington DC, 20233 \\ ${ }^{2}$ Fors Marsh Group, 1010 N Glebe Rd, Suite 510, Arlington, VA, 22201 \\ ${ }^{3}$ School of Psychology, Georgia Institute of Technology, 654 Cherry St., Atlanta, GA 30332 \\ Erica.L.Olmsted. Hawala@census.gov, \\ JBergstromaforsmarshgroup.com, Wendy@gatech.edu
}

\begin{abstract}
In a usability study on a portion of the Census Bureau Web site, we assess how people of different ages search for specific information by evaluating performance and strategy differences between age groups. We collected usability metrics of accuracy and efficiency, including mouse click data. Eye-tracking data were also collected including eye-movement patterns in pre-defined areas of interest. This paper focuses on the number of fixations (whether participants looked at the correct area of the screen) and number of unique visits (whether participants re-checked their answers). Results show that on the hard task only, older adults took longer to make the first click when initially starting the task, and there was a trend for younger adults to have higher accuracy. All age groups re-checked their answers suggesting that users of all ages experience difficulties when reading and comprehending complex data tables.
\end{abstract}

Keywords: eye tracking, usability, age differences, search strategy, mouse clicks, cognition.

\section{Introduction/Background}

Older adults are using the Internet more and more [1, 2, 3, 4]. In fact, since 2005, the largest increase in Internet use has been with adults aged 70 to 75 [5]. The Internet is useful and has potential to help older adults, (e.g., as a source of information, education, social support), yet at the same time, older adults may experience information overload and challenges when trying to find pertinent information. There is some evidence that older adults have difficulty using the Internet $[6,7,8,9,10,11]$ and thus use it less frequently than younger adults [12]. Possible explanations for this phenomenon include general age-related differences in cognition [13] or differences in strategies [11]. At present, there is little empirical evidence about how older adults search for information on Web sites, and whether age-related differences between

\footnotetext{
* Disclaimer: This report is released to inform interested parties of research and to encourage discussion. Any views expressed on the methodological issues are those of the authors and not necessarily those of the U.S. Census Bureau.
} 
younger, middle-age, and older adults actually exist (or whether the age-related differences are a proxy for experience, rather than age per se). The purpose of the present study was to:

- Conduct a systematic and in-depth assessment of how people of different ages search a Web site for specific information

- Identify performance and strategy differences.

Our goal was to understand the nature of the difficulties people have in order to provide guidance for Web site design and training.

We examined the interactions of younger, middle-age, and older adults with a datarich Web site as they completed typical tasks that required finding information. We used eye-tracking technology to examine search strategies and assessed where participants first clicked to find information, how long it took them to make their first click, how many clicks it took them to complete the task, and overall performance (i.e., accuracy, efficiency). We also noted what participants did after they initially found the "correct" answer-whether they immediately said they found their answer or whether they re-checked their answer before telling the test administrator the answer.

There is evidence of a speed/accuracy trade off among older adults when working on tasks that involve information processing [14, 15], reaction times [16, 17], and working memory [18]. Recent research also suggests that older adults may take longer to find target information in visual search tasks because they re-check their answers more often than younger adults [19]; this might translate to Internet searches as well. As Internet activity becomes ubiquitous, it is important to evaluate the effects of the speed/accuracy trade off among older adults during Web site interaction.

Based on established empirical research showing the speed/accuracy trade-off for older adults, we hypothesized that: (1) older adults would take longer to make their first click, but they would be more precise in their clicks; thus, they would have fewer clicks that would get them to the correct answer compared to other age groups; (2) younger adults would be more efficient than other age groups, but they would click often and freely, using "forward and backtrack" clicking behavior as they attempted to find the answer; 3 ) older adults would do more visual re-checking on the target or "answer" Web page than younger and middle-age adults.

\section{Methods}

\subsection{Participants}

Participant demographics are presented in Table 1. We tested three age groups, intentionally selecting age ranges that were far enough apart to detect age-related differences. Participants were residents of the metropolitan Washington DC area and were recruited via advertisements in local newspapers or through a database that is maintained by the US Census Bureau's Human Factors and Usability Research Group. Participants were given a $\$ 40$ honorarium. Participants completed a questionnaire about their computer use and Internet experience as well as questions that measured cognition. They also completed a questionnaire about their search and navigation habits and strategies. All participants reported being experienced with computers and 
the Internet but unfamiliar with the Web site used in this study, which was the American FactFinder (AFF). There was no age-related difference in reported difficulty in using the Internet, but older adults reported greater difficulty in learning to use new Web sites, compared to younger and middle-age adults. Groups did not differ in working memory (WAIS-III Backward Digit Span) [20] or verbal ability (Shipley Vocabulary) [21]; they differed in processing speed (WAIS-III Digit Symbol Coding), such that older adults scored lower than younger and middle-age adults, who did not differ from each other. See Table 1 for demographics and cognition scores.

Table 1. Mean (and Range) Demographics by Age Group

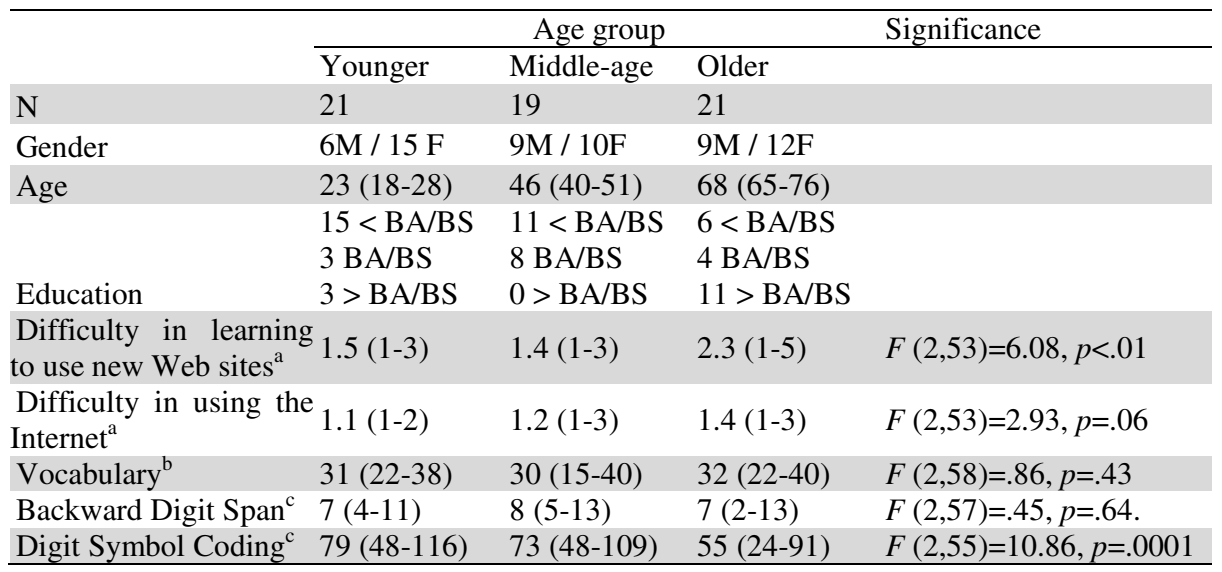

${ }^{\text {a }}$ Scale: 1 (Not difficult at all) - 5 (Extremely difficult)

${ }^{\mathrm{b}}$ Shipley Institute of Living Scale (Shipley, 1986)

${ }^{\mathrm{c}}$ WAIS-III, Wechsler Adult Intelligence Scale, 3rd Edition

\subsection{Procedure}

Sessions took place in the Human Factors and Usability Research Group's laboratory at the Census Bureau Headquarters. Prior to their arrival at the Census Bureau, each participant completed a set of cognitive battery tests over the phone. Each participant sat in a 10 ' $x 12$ ' room, facing a one-way mirror and a wall camera, in front of a 17'" LCD Tobii T-120 eye-tracking monitor.

After the test administrator (TA) read a brief introduction, the participant, working one-on-one with the TA, signed a consent form and completed the Digit Symbol Coding task and a brief calibration of their eyes by following a red circle as it moved on the screen. Then the TA left the room-the participant and the TA sat in separate rooms during the remainder of the session and communicated via microphones and speakers. The TA began video recording from the opposite side of the one-way mirror. The participant completed a short questionnaire about their Internet searching habits and strategies and the Shipley Vocabulary test [21], and then the participant was ready to begin finding information using the AFF Web site.

Participants read each task aloud, then used the Web site to locate the information (while working silently), and they stated their answer aloud when they felt they had 
found the correct answer. During each task, participants' eye movements were recorded unobtrusively. At the conclusion of the study, participants completed a final satisfaction questionnaire and answered debriefing questions from the TA.

Participants worked on five pre-determined information-seeking tasks on the legacy $^{1}$ AFF Web site. AFF is the Census Bureau's primary data dissemination Web site about the population, housing, and economy of the United States (see Figure 1). Participants completed typical tasks for general users of the Web site, and they completed tasks in the order of easiest to hardest to accomplish (determined by the complexity and number of steps needed to find the information). The first two tasks we categorized as easy; they required 2 and 3 steps, respectively, and the final three tasks we categorized as hard; they required 4, 4, and 6 steps, respectively. In this paper, we examine performance on the easiest and the hardest task. For the easiest task (Task 1: How many people live in Maryland?), participants could find the information via three different routes from the home page, and they needed a single number to accurately identify the answer. For the hardest task (Task 5: You are working on a project that involves work environments in the US, and you are interested in the history of coal mining. How many coal mining companies were in the US in 2007?), it was not clear from the home page where to begin, and the route to the answer did not follow an obvious path.

\subsection{Usability Metrics}

We assessed two typical usability metrics: accuracy and efficiency. For our dependent measures, accuracy was calculated as the percent of users who successfully completed the task, and efficiency was calculated in three ways: (1) the mean time to complete the task (successful completions only), (2) the mean time to make the first click, and (3) the mean number of clicks to complete the task (successful completions only).

Three independent coders (employed by the Census Bureau, but not involved with this project) were randomly assigned to conduct mouse-click coding. Each coder viewed video recordings and recorded the time to first click and number of clicks made per task. If the total number of clicks exceeded 31 clicks, the coders were instructed to stop counting.

\subsection{Eye Tracking}

Tobii Studio [22] recorded the eye-tracking data, and we examined eye-movement patterns in predefined areas of interest (AOIs). For Task 1, the AOIs were the three acceptable first clicks that would lead participants to the correct information (shown in Figure 1). On the "target" or answer pages, the AOI was the correct answer. For Task 1, the target answer was on three different pages, so we identified the AOI on each page. For Task 5, the AOI was the correct answer, which was also on three different pages, and thus, we identified AOIs on each of those pages.

${ }^{1}$ In 2012, a new American FactFinder (AFF) was released. The present study was conducted on the earlier AFF site, which is no longer available online and is referred to as the "legacy" version. 
For each AOI, we examined the total number of fixations to assess if participants looked at the correct area of the screen, and we examined the total number of unique visits to assess if participants re-checked the correct answer before deciding it was correct. See Figure 1.

\section{Results}

We asked the following questions when participants interacted with the Census Bureau's dense, data-rich Web site:

1. Were there age-related differences in performance (task accuracy; efficiency)?

2. When initially seeing the home page (i.e., on the first task), how much time elapsed before participants clicked on the correct click?

3. How many clicks did it take participants in the easiest and hardest task to find the answer they were looking for?

4. When honing in on an answer, how often did participants re-check their answer before finalizing their answer? Were there age-related differences in re-checking behavior?

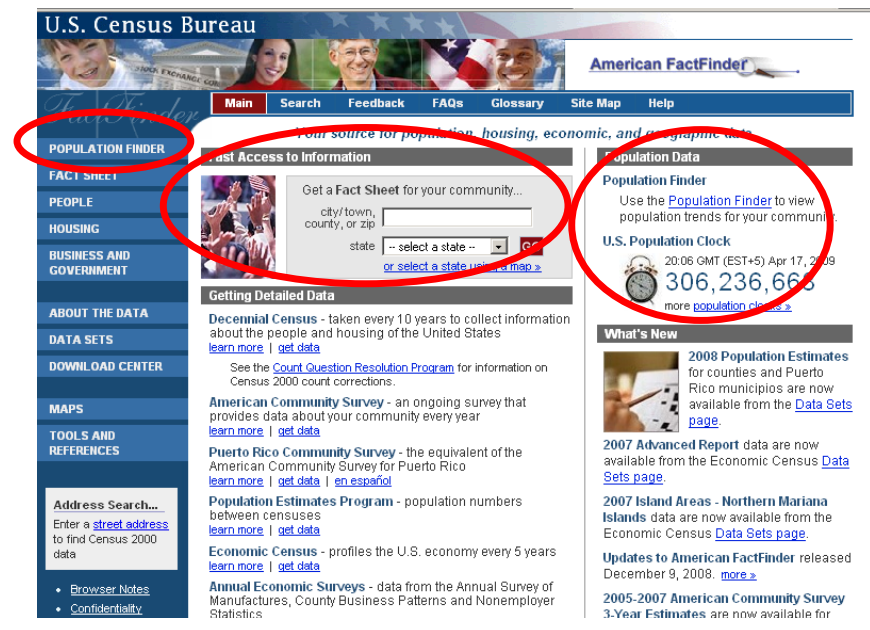

Fig. 1. AFF Web site home page. Areas of Interest (AOIs) for Task 1 are circled.

\subsection{Usability Metrics}

First we examined accuracy. We conducted one-way ANOVAs comparing age group. While this study was a convenience sample, done with participants in the DC area, in order to apply this analysis we are going to assume that this is a random sample of the population. We found that for Task 1 - the first, easiest task in the study-there was no age-related difference $(p=.70)$. For task 5 - the final and hardest task- there was a trend for younger adults to have higher accuracy than middle-age and older adults, $F(2,58)=2.66, p=.08$. 
Next we examined efficiency. For Task 1, we found no age-related difference in time to complete task $(p=.70)$ and number of clicks before finding the target answer $(p=.62)$. There also was no age-related difference in time to first click $(p=.37)$ for all participants, successful or not. For Task 5, for successful completions only, older adults took significantly longer than younger and middle-age adults to make the first click when starting the task, $F(2,53)=8.35, p<.001$. There was no age-related difference in time to complete the task, $(p=.71)$ or in the total number of clicks before finding the target answer $(p=.20)$. See Table 2 .

Thus, it appears that older adults do not differ in performance when working on an easy task; however, when working on a hard task, age related differences emerge. Older adults have greater difficulties, as seen in the increased time to initially make the first click and lower accuracy.

Table 2. Mean (and SD) Performance by Age Group

\begin{tabular}{|c|c|c|c|c|}
\hline & \multicolumn{3}{|c|}{ Age group } & \multirow[t]{2}{*}{ Significance } \\
\hline & Younger & Middle-age & Older & \\
\hline \multicolumn{5}{|l|}{ Task 1 (easy) } \\
\hline $\begin{array}{l}\text { Accuracy } \\
\text { (\% complete) }\end{array}$ & $71 \%(46 \%)$ & $79 \%(41 \%)$ & $\begin{array}{l}67 \% \\
(48 \%)\end{array}$ & $F(2,58)=.36, p=.70$ \\
\hline Seconds to first click & $\begin{array}{l}23.20 \\
(15.65)\end{array}$ & $\begin{array}{l}25.25 \\
(24.01)\end{array}$ & $\begin{array}{l}31.53 \\
(19.00)\end{array}$ & $F(2,54)=1.01, p=.37$ \\
\hline $\begin{array}{l}\text { Seconds to complete } \\
\text { task (successes only) }\end{array}$ & $\begin{array}{l}95.07 \\
(79.75)\end{array}$ & $\begin{array}{l}127.57 \\
(127.33)\end{array}$ & $\begin{array}{l}127.43 \\
(132.46)\end{array}$ & $F(2,39)=.37, p=.70$ \\
\hline $\begin{array}{l}\text { Number of } \\
\text { clicks to complete } \\
\text { (successes only) }\end{array}$ & $7.73(7.92)$ & $6.75(3.91)$ & $5.43(6.02)$ & $F(2,38)=.48, p=.62$ \\
\hline \multicolumn{5}{|l|}{ Task 5 (hard) } \\
\hline $\begin{array}{l}\text { Accuracy } \\
\text { (\% complete) }\end{array}$ & $70 \%(46 \%)$ & $37 \%(50 \%)$ & $\begin{array}{l}43 \% \\
(51 \%)\end{array}$ & $F(2,58)=2.66, p=.08$ \\
\hline Seconds to first click & $\begin{array}{l}19.62 \\
(11.38)\end{array}$ & $\begin{array}{l}25.09 \\
(14.99)\end{array}$ & $\begin{array}{c}48.80 \\
(35.70)\end{array}$ & $F(2,53)=8.35, p<.001$ \\
\hline $\begin{array}{l}\text { Seconds to complete } \\
\text { task (successes only) }\end{array}$ & $\begin{array}{l}176.54 \\
(132.05)\end{array}$ & $\begin{array}{l}165.86 \\
(135.46)\end{array}$ & $\begin{array}{l}234 \\
(64.95)\end{array}$ & $F(2,26)=.35, p=.71$ \\
\hline $\begin{array}{l}\text { Number of } \\
\text { clicks to complete } \\
\text { (successes only) }\end{array}$ & $12.39(9.96)$ & $5.72(1.77)$ & $8.25(5.45)$ & $F(2,24)=1.74, p=.20$ \\
\hline
\end{tabular}

\subsection{Eye Tracking}

First we examined participants' initial interaction with the Web site-that is, on Task 1, when the session began. For Task 1, on the home page, there was no significant age-related difference in the total number of fixations for the correct click AOIs (the clicks that participants could make to successfully complete the task) $(p=.40)$. Consistent with the fixation data, there was no age-related difference in re-checking to those AOIs, (e.g., total unique visits) $(p=.52)$. Thus, for the first task (which was the easiest one), there was no age-related difference in where participants looked and the number of times they re-checked the AOIs before making a click. See Table 3.

Next we examined eye-tracking behavior on the answer pages for participants who successfully completed the tasks. For Task 1, there was no significant age-related difference in the total number of fixations on the correct answer AOI $(p=.69)$. 
Consistent with the fixation data, there was no age-related difference in re-checking to those AOIs, $(p=.62)$.

For Task 5, the hardest task, we examined performance on the answer page only (for successful completions only). There was no age-related difference in total number of fixations on the correct answer AOIs $(p=.98)$ and no significant age-related difference in re-checking to those AOIs $(p=.99)$. However, note that these data are for fixations once the correct page was reached for successful completions only and may represent the "best" middle-aged and older adult searchers.

To summarize, as seen in Table 3, for both Task 1 all age groups re-checked that their first click was correct before making it, and for both Task 1 and Task 5, all age groups re-checked their answer before telling the TA what the correct answer was.

Table 3. Mean (and SD) Eye-Tracking Data by Age Group

\begin{tabular}{|c|c|c|c|c|}
\hline & \multicolumn{3}{|c|}{ Age group } & \multirow[t]{2}{*}{ Significance } \\
\hline & Younger & Middle-age & Older & \\
\hline \multicolumn{5}{|l|}{ Task 1 (easy) } \\
\hline $\begin{array}{l}\text { Total fixations: correct } \\
\text { click AOI- home pg }\end{array}$ & $\begin{array}{l}10.48 \\
(10.45)\end{array}$ & $\begin{array}{l}13.11 \\
(17.49)\end{array}$ & $\begin{array}{l}28.86 \\
(6.45)\end{array}$ & $F(2,57)=.94, p=.40$ \\
\hline $\begin{array}{l}\text { Total re-checking: } \\
\text { correct click AOI- } \\
\text { home pg }\end{array}$ & $\begin{array}{l}8.14 \\
(4.79)\end{array}$ & $\begin{array}{l}9.32 \\
(8.62)\end{array}$ & $\begin{array}{l}11.20 \\
(11.25)\end{array}$ & $F(2,57)=.69, p=.52$ \\
\hline $\begin{array}{l}\text { Total fixations: correct } \\
\text { click AOI- answer pg* }\end{array}$ & $\begin{array}{l}10.27 \\
(10.24)\end{array}$ & $\begin{array}{l}9.60 \\
(10.06)\end{array}$ & $\begin{array}{l}7.29 \\
(8.66)\end{array}$ & $F(2,41)=.37, p=.69$ \\
\hline $\begin{array}{l}\text { Total re-checking: } \\
\text { correct click AOI- } \\
\text { answer pg* }\end{array}$ & $\begin{array}{l}8.20 \\
(7.90)\end{array}$ & $\begin{array}{l}8.53 \\
(9.31)\end{array}$ & $\begin{array}{l}5.86 \\
(6.06)\end{array}$ & $F(2,41)=.49, p=.62$ \\
\hline \multicolumn{5}{|l|}{ Task 5 (hard) } \\
\hline $\begin{array}{l}\text { Total fixations: correct } \\
\text { click AOI- answer pg* }\end{array}$ & $\begin{array}{l}4.64 \\
(4.20)\end{array}$ & $\begin{array}{l}4.86 \\
(2.91)\end{array}$ & $\begin{array}{l}4.44 \\
(5.18)\end{array}$ & $F(2,27)=.02, p=.98$ \\
\hline $\begin{array}{l}\text { Total re-checking: } \\
\text { correct click AOI- } \\
\text { answer pg* }\end{array}$ & $\begin{array}{l}4.57 \\
(4.07)\end{array}$ & $\begin{array}{l}4.43 \\
(3.41)\end{array}$ & $\begin{array}{l}4.33 \\
(5.17)\end{array}$ & $F(2,27)=.01, p=.99$ \\
\hline
\end{tabular}

* Successful completions only.

\section{Conclusions}

On the first simple search task-where participants looked for a single piece of information-older adults were as accurate and efficient as younger and middle-age adults. As well, when first looking at the home page, while attempting to answer the first task, there were no differences in how long it took before participants made their first click or in the number of clicks made before arriving at the target answer page.

However, differences in performance were visible on some of the measures with the hardest task where participants had to find content deeper in the site, on a path that was not straightforward. This is in line with previous research [23, 24, 25] that shows that with a more cognitively challenging task, age-related differences are apparent.

The pattern of results appear to support two of our hypotheses-that (1) older adults would take longer to make their first click, but they would be more precise in their clicks; thus, they would have fewer clicks that would get them to the correct 
answer compared to other age groups, and (2) younger adults would be more efficient than other age groups, but they would click often and freely, using "forward and backtrack" clicking behavior as they attempted to find the answer.

While not statistically significant, the finding that older adults use fewer clicks to find the correct target or answer page but still take longer to make the first click, and they take longer overall is in line with research that shows a speed/accuracy trade-off with older adults. Our findings for the hard task are consistent with this literature. As well, we see that younger adults are more apt to click on a link and backtrack as they go about honing in on the target answer page. It is possible that with a larger sample size, the click data could be significant; future research should examine this.

It is important to note that the older adults in this study were high functioning - as seen in Table 1, they were educated and did not differ from the younger adults on vocabulary or working memory. There were age-related differences in perceptual speed, which might contribute to the longer search times for the first exposure to the Web site. Future efforts should include a broader range of participants to assess cognitive predictors of successful searching.

Many of our measures were on successful completions only (e.g., examining eye tracking on answer pages), where for Task 5, nine older adults, 11 middle-age adults, and 17 younger adults made it to the correct answer page. Thus the comparisons of eye-tracking performance for the successful searchers is only a subset of the middleage and older searchers. We are currently conducting additional analyses on the eye-tracking patterns for unsuccessful searchers to understand their performance difficulties. The fact that only some older adults made it to the end of this difficult task implies that they may be higher functioning than average older adults.

This study found that there were no differences in re-checking behavior for successful searchers. It is interesting that when participants had to search for a piece of information on a data display table, with distractors on the results page, all age groups re-checked their answer. When confronted with a complex, data rich Web site, it appears that all users experience challenges in reading a data table as all adults in this study re-checked the number they were looking at before responding with their answer. Putting complex data tables online appears to cause usability issues for all age groups in that they all re-check their answers. In the future, online mediums should consider new ways to visualize complex data. Instead of what has been the norm of putting paper tables online, data visualization research could offer alternatives to access and aid in interpreting data online. For example, future research could consider if a simplified interface, with reduced distractors and access to widgets that allow the user to control the visual input may make a difference for users when honing in on an answer.

\section{References}

1. O'Connell, T.A.: The why and how of senior-focused design. In: Lazar, J. (ed.) Universal Usability: Designing Computer Interfaces for Diverse Users, pp. 43-92. Wiley, West Sus$\operatorname{sex}(2007)$

2. US Census Bureau: Computer and Internet Use in the United States: 2003 (2005), http: //www. census.gov/hhes/computer/publications / 2003 .html 
3. US Census Bureau: Reported Internet Usage for Households, by Selected House-holder Characteristics: 2009. Source: Current Population Survey, US Census Bureau (2010a)

4. US Census Bureau: Computer and Internet Use in the United States: 2010 (2010b), http: / /www. census.gov/population/www/socdemo/computer.html

5. Pew Internet \& American Life Project (2009), http: / / pewinternet.org/Reports/2010/Generations-2010.aspx

6. Chadwick-Dias, A., McNulty, M., Tullis, T.: Web usability and age: How design changes can improve performance. In: Proceedings of the Conference on Universal Usability, Vancouver, British Columbia, Canada (November 2003)

7. Czaja, S., Lee, C.: The Internet and older adults: Design challenges and opportunities. In: Charness, N., Parks, D., Sabel, B. (eds.) Communication, Technology and Aging: Opportunities and Challenges, pp. 60-78. Springer, New York (2001)

8. Pernice, K., Nielsen, J.: Web Usability for Senior Citizens. Design Guidelines Based on Usability Studies with People Age 65 and Older. Nielsen Norman Group, Fremont (2002)

9. Redish, J.C., Chisnell, D.: Designing web sites for older adults: A review of recent research (2004), AARP

http: / /assets.aarp.org/www. aarp.org_/articles/research/ oww/AARP-LitReview2004.pdf

10. Rogers, W.A., Badre, A.: The Web user: Older adults. In: Badre, A. (ed.) Shaping Web Usability: Interaction Design in Context, pp. 91-108. Addison-Wesley, Boston (2002)

11. Stronge, A., Rogers, W., Fisk, A.: Web-based information search: effects of strategy use and age on search success. Human Factors 48, 434-446 (2006)

12. Olson, K.A., O’Brien, M.A., Rogers, W.A., Charness, N.: Diffusion of technology: Frequency of use for younger and older adults. Ageing International 36, 123-145 (2011)

13. Fisk, A.D., Rogers, W.A., Charness, N., Czaja, S.J., Sharit, J.: Designing for older adults: Principles and creative human factors approaches, 2nd edn. CRC Press, Boca Raton (2009)

14. Brébion, G.: Language Processing, Slowing, and Speed/Accuracy Trade-Off in the Elderly. Experimental Aging Research 27(2), 137-150 (2001)

15. Strayer, D., Wickens, C., Braune, R.: Adult age differences in the speed and capacity of information processing: II An electrophysiological approach. Psychology and Aging 2(2), 99-110 (1987)

16. Howard, J.H., Howard, D.V., Dennis, N.A., Yankovich, H.: Event Timing and Age Deficits in Higher-Order Sequence Learning. Aging, Neuropsychology, and Cognitio 14(6), 647-668 (2007)

17. Salthouse, T.: Adult age and the speed-accuracy trade-off. Ergonomic 22(7), 811-821 (1979)

18. Brébion, G.: Working Memory, Language Comprehension, and Aging: Four Experiments to Understand the Deficit. Experimental Aging Research 29(3), 269-301 (2003)

19. Mitzner, T.L., Touron, D.R., Rogers, W.A., Hertzog, C.: Checking it Twice: Age-related Differences in Double Checking during Visual Search. Proceedings of the Human Factors and Ergonomics Society Annual Meeting 54(18), 1326-1330 (2010)

20. Wechsler, D.: Wechsler adult intelligence scale (WAIS-III), 3rd edn. The Psychological Corporation, New York (1997)

21. Shipley, W.: Shipley Institute of Living Scale. Western Psychological Press, Los Angeles (1986)

22. Tobii Studio version 3.1.3.6615-RC (2012), http://www.tobii.com/en/eyetracking-research/global/ 
23. Plude, D.J., Doussard-Roosevelt, J.A.: Aging, selective attention, and feature integration. Psychology and Aging 4(1), 98-105 (1989)

24. Craik, F.I.M., Byrd, M.: Aging and cognitive deficits: The role of attentional resources. In: Craik, F.I.M., Trehub, S.E. (eds.) Aging and Cognitive Processes, pp. 191-211. Plenum, New York (1982)

25. Grahame, M., Laberge, J., Sciafla, C.T.: Age differences in search of Web pages: The effects of link size, link number, and clutter. Human Factors: The Journal of the Human Factors and Ergonomics Society 46(3), 385-398 (2004) 